\title{
Evaluating Machine Learning Models for the Fast Identification of Contingency Cases
}

\author{
Florian Schaefer ${ }^{1}$, Jan-Hendrik Menke ${ }^{1}$, and Martin Braun ${ }^{1}$ \\ ${ }^{1}$ University of Kassel
}

August 26, 2020

\begin{abstract}
Fast approximations of power flow results are beneficial in power system planning and live operation. In planning, millions of power flow calculations are necessary if multiple years, different control strategies, or contingency policies are to be considered. In live operation, grid operators must assess if grid states comply with contingency requirements in a short time. In this paper, we compare regression and classification methods to either predict multi-variable results, e.g., bus voltage magnitudes and line loadings, or binary classifications of time steps to identify critical loading situations. We test the methods on three realistic power systems based on time series in $15 \mathrm{~min}$ and $5 \mathrm{~min}$ resolution of one year. We compare different machine learning models, such as multilayer perceptrons (MLPs), decision trees, k-nearest neighbors, gradient boosting, and evaluate the required training time and prediction times as well as the prediction errors. We additionally determine the amount of training data needed for each method and show results, including the approximation of untrained curtailment of generation. Regarding the compared methods, we identified the MLPs as most suitable for the task. The MLP-based models can predict critical situations with an accuracy of $97-98 \%$ and a very low number of false negative predictions of $0.0-0.64 \%$.
\end{abstract}

\section{Hosted file}

25_08_2020_wiley.pdf available at https://authorea.com/users/354105/articles/477755evaluating-machine-learning-models-for-the-fast-identification-of-contingency-cases 